\title{
Sound transmission between absorbing parallel planes
}

\author{
C. J. Hurst \\ Department of Mechanical Engineering, Virginia Polytechnic Institute and State University, Blacksburg, \\ Virginia 24061
}

(Received 5 September 1978; accepted for publication 28 September 1979)

\begin{abstract}
Partially absorbing parallel surfaces can be the dominant acoustical feature of many rooms. The sound fields in such rooms are not diffuse, which causes difficulty in predicting sound pressure levels. A method is developed for predicting sound pressure levels in these rooms. It rests on the prediction of sound pressure levels caused by a nondirectional source of known sound power radiating between absorbing parallel planes. The development proceeds from a geometrical acoustics viewpoint. Good correlation has been found between predicted and measured levels in existing rooms having simple geometries.
\end{abstract}

PACS numbers: 43.55.Br, 43.55.Ka, 43.20.Dk

\section{INTRODUCTION}

The acoustics of large rooms has been a topic of concern since the founding of the Acoustical Society. Despite considerable work since then, there remain many situations in which it is difficult or impossible to predict the sound pressure level inside closed spaces. The work reported in this paper is the outgrowth of an attempt to use geometric acoustics to predict sound pressure levels in large rooms with low ceilings, such as are often found in factories, open plan offices, and the like.

Geometric acoustics concepts have often been used to discuss the sound transmission in rooms. The basic reverberation time expressions of Millington ${ }^{1}$ and Eyring, ${ }^{2}$ for example, were derived using this approach. Geometric acoustics concepts, and the analogy with light also have been used in the design of auditoria. ${ }^{3}$ The classical approach to the prediction of sound pressures in large enclosures has been to reason from geometric acoustics concepts to the point of assuming a diffuse sound field in a room. This assumption has been shown to hold so long as room dimensions do not exceed about $1: 1.5: 2$, or so long as all wall absorption coefficients are nearly the same. ${ }^{4}$ If these conditions are not met, the results arrived at from assuming a diffuse field to exist may be significantly in error.

The advent of the high-speed digital computer has made possible the prediction of sound pressure levels inside of enclosures which do not meet the diffuse field requirements. ${ }^{5-8}$ To do this, it is necessary to predict the significant sound paths from a source to a receiver. Once these paths have been found it is only necessary to determine the absorption of each surface the sound ray strikes, and to sum all of the energy contributions arriving at the measuring point. The disadvantage of this approach is that there may be a great number of important sound paths to be found in any real (complex) room. Thus, considerable computing power and computing time may be required using geometric acoustics concepts. It has been found that many large rooms have one or more sets of plane surfaces as boundaries which are relatively close together. Under these conditions, it has been shown that the reflected sound paths between the closely spaced parallel walls can be the dominant acoustical feature. ${ }^{7}$ This type of reflection path is easily handled, and forms the basis for this paper. The equations for sound transmission between parallel, partially absorbing planes will be developed, and examples of how the results may be applied in irregular rooms will be given.

Galaitsis and Patterson have recently published an analysis very similar to this ${ }^{9}$; their investigation was prompted by the necessity of predicting sound in mines. Once again, the dominant acoustical feature was the closely spaced parallel planes. The primary difference between this work and the work of Galaitsis and Patterson is that this work includes the possibility that the two parallel planes may have different absorption coefficients. Galaitsis and Patterson investigated this situation for planes having the same acoustic absorption. Also, the results in this investigation are presented in what is believed to be a more readily usable form for computation than those of Galaitsis and Patterson.

\section{DEVELOPMENT OF THE EQUATIONS}

There are three distinct steps in the derivation of the equations for sound transmission between parallel absorbing planes. First, the geometric equations for the sound ray paths between the source and the receiver must be obtained. Then the reduction in intensity along each ray path because of spherical spreading and absorption by the planes must be incorporated. Finally, the energy arriving at the receiver along each of the ray paths must be combined to determine the net sound pressure level.

The geometry of the ray paths can be determined with the aid of Figs. 1 and 2. The only assumption needed is that the angle of reflection at a boundary is equal to the angle of incidence. Figure 1 shows the simplest reflected rays; those undergoing only one reflection. The ray paths SAR and SBR can most easily be determined by using the "image sources" $S_{1}^{\prime}$ and $S_{2}^{\prime}$. These locations can be found by successive rotations of the figure around the lines representing plane 1 and plane 2. The parameter of interest in this study is the ray path length. Path length $S A R$ can easily be found, as it is equal to the path length $S_{1}^{\prime} A R$. The Pythagorean Theorem gives for the path $S_{1}^{\prime} A R$

$$
\overline{(S A R)}=\left[(\Delta x)^{2}+\left(y_{S}-y_{R}\right)^{2}\right]^{1 / 2} \text {. }
$$




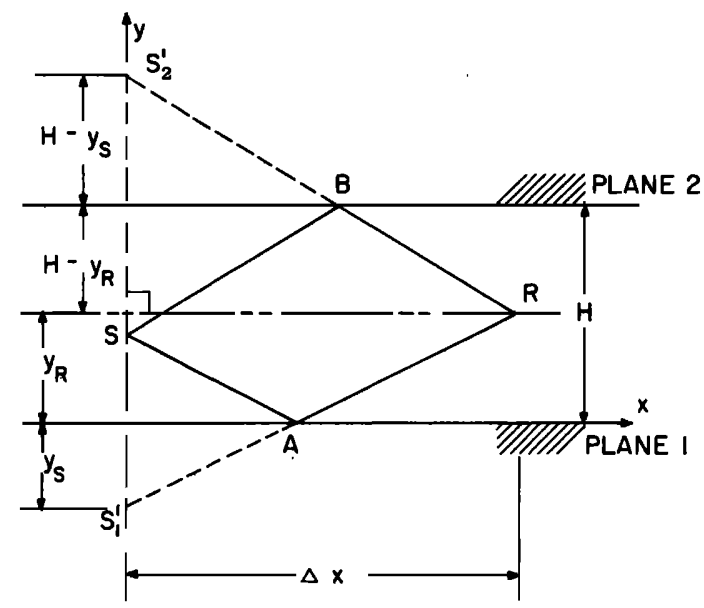

FIG. 1. Geometry for single reflection sound paths.

For path $S B R$ (or $S_{2}^{\prime} B R$ ) the length is

$$
(\overline{S B R})=\left\{(\Delta x)^{2}+\left[2 H-\left(y_{S}+y_{R}\right)\right]^{2}\right\}^{1 / 2} \text {. }
$$

A similar procedure is followed for cases involving higher numbers of reflections. Path $S A B R$ in Fig. 2 has the length

$$
(\overline{S A B R})=\left\{(\Delta x)^{2}+\left[2 H-\left(y_{R}-y_{s}\right)\right]^{2}\right\}^{1 / 2},
$$

and path $S C D R$ has the length

$$
(\overline{S C D R})=\left\{(\Delta x)^{2}+\left[2 H+\left(y_{R}-y_{S}\right)\right]^{2}\right\}^{1 / 2} \text {. }
$$

This procedure is followed for succeedingly higher numbers of reflections. There turn out to be four cases which must be considered. Separate consideration must be given to ray paths involving even or odd numbers of reflections from the walls. Rays undergoing even or odd numbers of reflections must be further categorized between those which strike the upper plane first and those which strike the lower plane first. The equations

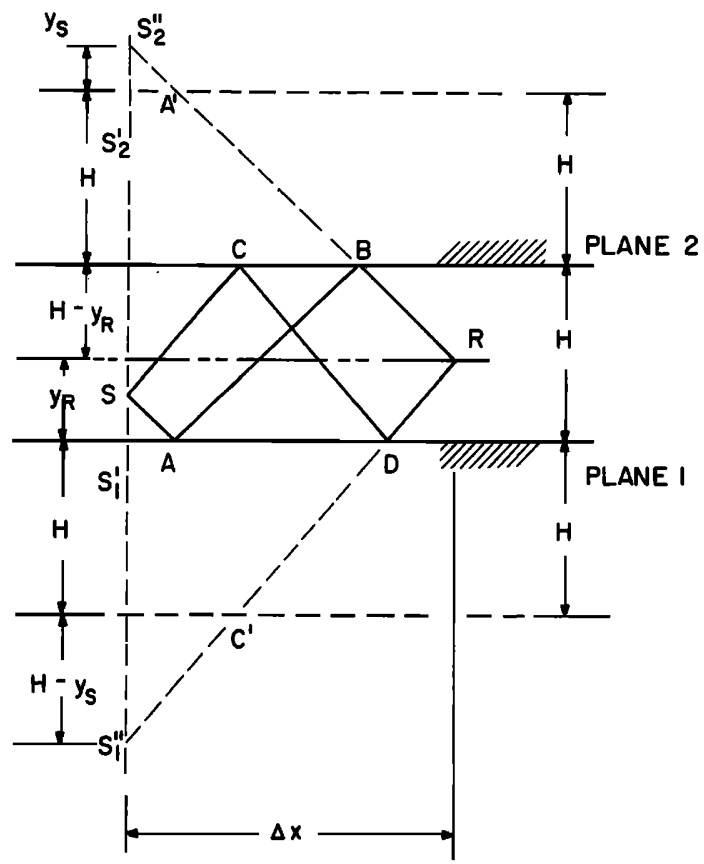

FIG. 2. Geometry for two reflection sound paths. can also be written in terms of the number of reflections along the path. The equations are as follows:

For the odd number $i$ of reflections the lengths $l$ are given by

$l_{i, 1}^{2}=(\Delta x)^{2}+\left[i H+\left(y_{S}+y_{R}-H\right)\right]^{2}, \quad$ (lower wall first),

$l_{i, 3}^{2}=(\Delta x)^{2}+\left[i H-\left(y_{S}+y_{R}-H\right)\right]^{2}, \quad$ (upper wall first).

For the even number $j$ of reflections the lengths $l$ are given by

$l_{j, 2}^{2}=(\Delta x)^{2}+\left[j H-\left(y_{R}-y_{S}\right)\right]^{2}, \quad$ (lower wall first),

$l_{j, 4}^{2}=(\Delta x)^{2}+\left[j H+\left(y_{R}-y_{S}\right)\right]^{2}, \quad$ (upper wall first).

With the geometry problem solved, attention can next be turned to determining the reduction in intensity along each ray path.

Calculations for the reduction in intensity along a ray path rest on two basic assumptions. First, the intensity along each ray path is assumed to decrease because of spherical spreading of the sound energy. Secondly, all of the acoustic absorption is assumed to take place at the walls, and to be independent of the angle of incidence of the sound. In fact, absorption of most surfaces does vary with angle, but the process of summing over several ray paths (angles) has an averaging effect similar to that implicit in reverberation room determinations of the random-incidence absorption coefficients used in the calculation.

The acoustic energy arriving at a receiver location after undergoing reflections and absorptions along a given ray path can be determined with the aid of Fig. 3. If the acoustic source is emitting $W$ watts of acoustic power, the intensity arriving at the first reflection point $I_{1}$ is given by

$$
I_{1}=W / 4 \pi l_{1}^{2} \text {. }
$$

After reflection and absorption

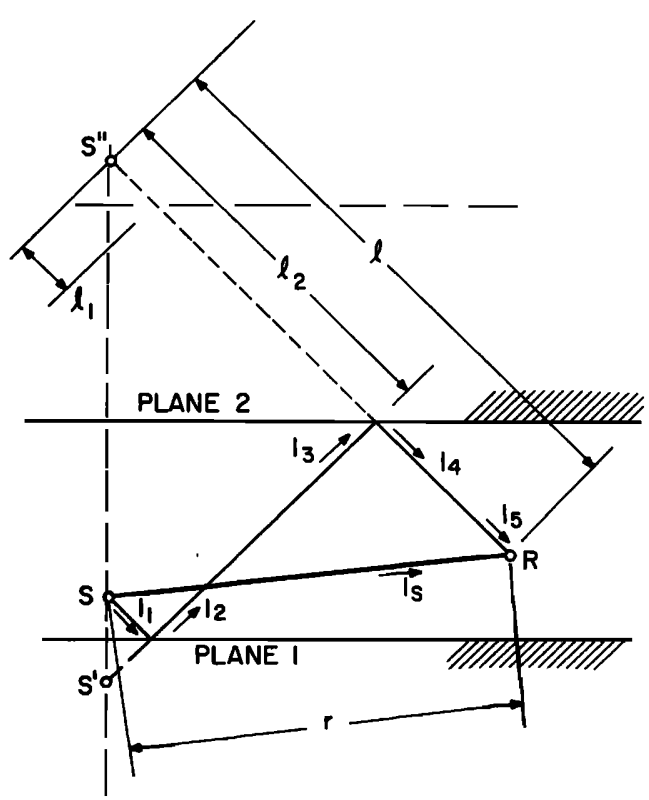

FIG. 3. Two reflection ray with intensities indicated along ray path. 


$$
I_{2}=I_{1}\left(1-\alpha_{1}\right)=\left(W / 4 \pi l_{1}^{2}\right)\left(1-\alpha_{1}\right) .
$$

This intensity is further reduced because of spherical spreading before the second reflection. The incident intensity for the second reflection is, therefore,

$$
I_{3}=I_{2}\left(l_{1}^{2} / l_{2}^{2}\right)=\left(W / 4 \pi l_{2}^{2}\right)\left(1-\alpha_{1}\right) .
$$

After reflection and absorption,

$$
I_{4}=I_{3}\left(1-\alpha_{2}\right)=\left(W / 4 \pi l_{2}^{2}\right)\left(1-\alpha_{1}\right)\left(1-\alpha_{2}\right) .
$$

At the receiver location, the result is

$$
I_{5}=I_{4}\left(l^{2} / l_{2}^{2}\right)=\left(W / 4 \pi l^{2}\right)\left(1-\alpha_{1}\right)\left(1-\alpha_{2}\right) .
$$

For convenience, this can be expressed in terms of $I_{s}$, the intensity at the receiver along the direct transmission path;

$$
I_{S}=W / 4 \pi R^{2} .
$$

The expression can also be revised to include $m$ reflections from plane 1 and $n$ reflections from plane 2 .

When these modifications are included,

$$
I_{5}=I_{S}\left(R^{2} / l^{2}\right)\left(1-\alpha_{1}\right)^{m}\left(1-\alpha_{2}\right)^{n} .
$$

This general equation can now be applied to each of the four reflection cases introduced above. For instance, the intensity along a path involving three reflections, with the lower plane being struck first, would be

$$
\begin{aligned}
I_{3,1} & =I_{S} \frac{R^{2}}{l_{3,1}^{2}}\left(1-\alpha_{1}\right)^{2}\left(1-\alpha_{2}\right) \\
& =I_{S} \frac{\left(y_{R}-y_{S}\right)^{2}+(\Delta x)^{2}}{(\Delta x)^{2}+\left[3 H+\left(y_{S}+y_{R}-H\right)\right]^{2}}\left(1-\alpha_{1}\right)^{2}\left(1-\alpha_{2}\right) .
\end{aligned}
$$

The final step in determining the sound pressure level at the receiver location is the summing of the acoustic energy arriving along each of the ray paths. Summing of intensities rather than pressures along each ray path was chosen because of uncertainties concerning the phasing of the arriving sound. In noise control work the source is often an incoherent one. Furthermore, the amount of phase-shift undergone at each reflection was difficult to specify for many acoustic materials, particularly given the variation with angle of incidence. The decision, therefore, was to use time averaged quantities and to sum intensities along the ray paths. When the summing is accomplished over all four cases involving reflections and the direct transmission ray from $S$ to $R$ is included, the resulting expression for intensity is;

$$
\begin{aligned}
\mathrm{I}= & \mathrm{I}_{S}\left[1+\sum_{i=1,3}^{\infty} R^{2}\left(\frac{\left(1-\alpha_{1}\right)^{(i-1) / 2}\left(1-\alpha_{2}\right)^{(i+1) / 2}}{l_{i, 3}^{2}}\right)\right. \\
& \left.+\frac{\left(1-\alpha_{1}\right)^{(i+1) / 2}\left(1-\alpha_{2}\right)^{(i-1) / 2}}{l_{i, 1}^{2}}\right) \\
& \left.+\sum_{\substack{j=2,4 \\
\text { (evenj } j . . .}}^{\infty} R^{2}\left(\left(1-\alpha_{1}\right)^{j / 2}\left(1-\alpha_{2}\right)^{j / 2} \frac{1}{l_{i, 2}^{2}}+\frac{1}{l_{i, 4}^{2}}\right)\right],
\end{aligned}
$$

where the lengths are given in Eqs. (5)-(8). If both sides of Eq. (17) are divided by the reference intensity $I_{0}$, it is possible to express this result in terms of intensity levels $L_{I}$,

$$
L_{I}-\left(L_{I}\right)_{\text {direct }}=10 \log (1+\text { SUM }) \text {. }
$$

It should be noted that each term in the sums shown in Eq. (17) is multiplied by the square of a length ratio. It is possible to rearrange each of these ratios so that all distances are expressed in nondimensional form as a multiple of the plane separation distance $H$. Thus,

$$
\begin{aligned}
\frac{R^{2}}{l_{i, 1}^{2}} & =\frac{R^{2}}{(\Delta x)^{2}+\left[i H+\left(y_{S}+y_{R}-H\right)\right]^{2}} \\
& =\frac{(R / H)^{2}}{(\Delta x / H)^{2}+\left[i+\left(y_{S} / H+y_{R} / H-1\right)\right]^{2}}, \\
\frac{R^{2}}{l_{i, 3}^{2}} & =\frac{(R / H)^{2}}{(\Delta x / H)^{2}+\left[i-\left(y_{S} / H+y_{R} / H-1\right)\right]^{2}}, \\
\frac{R^{2}}{l_{j, 2}^{2}} & =\frac{(R / H)^{2}}{(\Delta x / H)^{2}+\left[j-\left(y_{R} / H-y_{S} / H\right)\right]^{2}}, \\
\frac{R^{2}}{l_{j, 4}^{2}} & =\frac{(R / H)^{2}}{(\Delta x / H)^{2}+\left[j+\left(y_{R} / H-y_{S} / H\right)\right]^{2}} .
\end{aligned}
$$

Calculations based on Eqs. (17)-(22) can be carried out on a digital computer with ease. The series is strongly convergent, particularly as the absorption coefficient of either plane approaches unity. This is as it should be; Eq. (17) reduces to that for spherical spreading as the absorption of the planes approaches unity.

The SUM has been calculated for an arbitrarily chosen geometry, and the result is shown in Fig. 4. The dimensionless source and receiver height locations were chosen to be "typical" for a room if the plane spacing $H$ were $5 \mathrm{~m}$. A hard floor ( $\alpha=0.00$ Sabines) was assumed. The horizontal separation distance $\Delta x$ was varied to change $R$. Curves are shown for $\alpha_{2}$ ranging from 0.0 to 0.9 . The summation for each point was carried out until the succeeding term caused the result to change by less than $0.01 \mathrm{~dB}$.

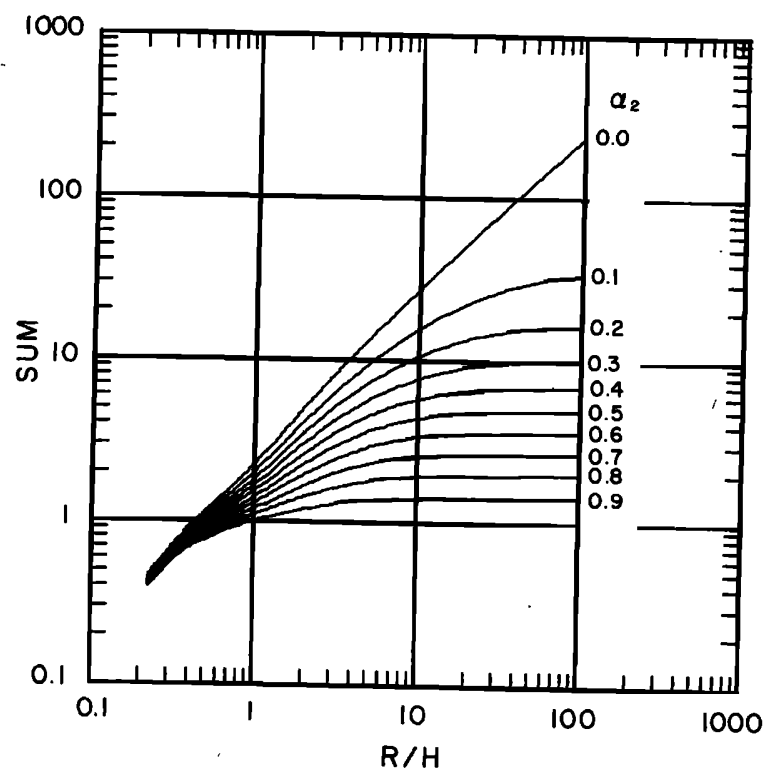

FIG. 4. Results of summation for one hard wall and one wall which ranges from hard to soft. $\alpha_{1}=0.0, y_{S} / H=0.1, y_{R} / H$ $=0.2$. 
Two things are apparent in Fig. 4. First, it is apparent that the absorption of plane 2 is not a major factor for $R / H$ values less than about 0.5 . It is also evident that the contribution of the reflected sound to the overall sound pressure level is less than that of the sound reaching the observer directly, since SUM is less than one in this region. The other thing that is apparent is that all of the curves except the $\alpha_{2}=0.0$ curve approach a fixed value asymptotically at the higher $R / H$ values. Each of these merits discussion.

The first area to be discussed will be the region where $R / H$ is less than one. For the source and receiver locations chosen (source near the lower wall, receiver $20 \%$ of the way up between the planes) it can be seen in Fig. 4 that the absorption of the upper plane is not an important factor in the result. Before concluding that acoustic absorption is unimportant in this region, however, further investigation is required. A second set of calculations for the SUM is shown in Fig. 5. This set of calculations was performed for slightly higher source and receiver locations, and for two different absorptions on the lower plane $\left(\alpha_{1}\right)$. The lower set of curves resulted from calculations using $\alpha_{1}$ of 0.8 , and the upper set of curves for an $\alpha_{1}$ of 0.2 . It will be seen that there is considerable variation in the results (nearly an order of magnitude) for $R / H$ less than about one. SUM must be added to one, however, before it is of any use in predicting the sound pressure level. Thus, what is important is not SUM, but $(1+$ SUM). When calculations are made on sound pressure levels for the most extreme cases shown in Figs. 4 and 5 (both absorptions equal to zero, and both absorptions equal to 0.8 ), the maximum difference in sound pressure level may be found to be about $4 \mathrm{~dB}$ at an $R / H$ of one. However, if the same calculation is performed at

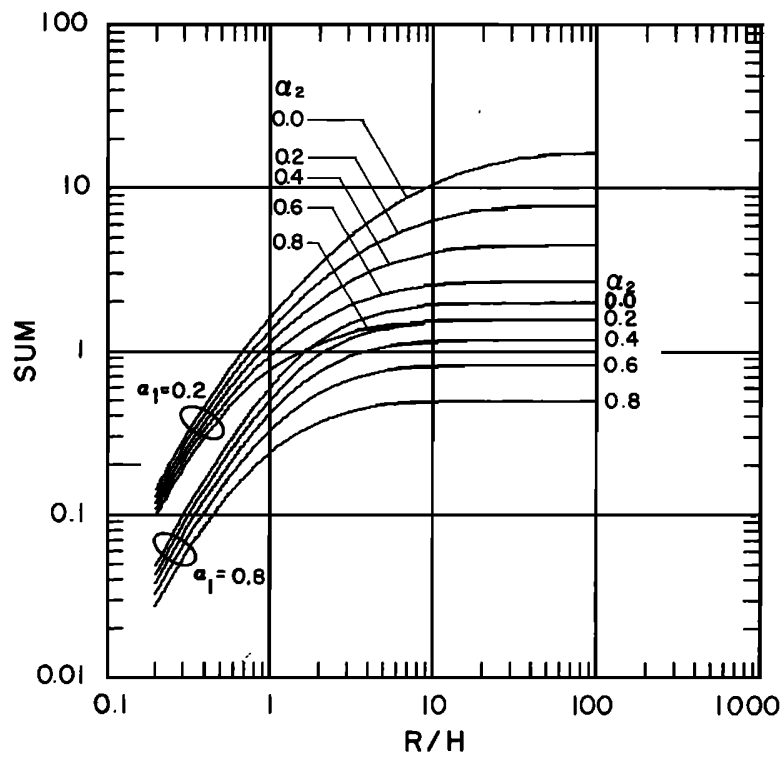

FIG. 5. Results of summation for different plane absorptions. For set 1, $\alpha_{1}=0.2$. For set $2, \alpha_{1}=0.8$. $y_{S} / H=0.2, y_{R} / H$ $=0.4$. an $R / H$ value of 0.2 , the difference will be found to be about $1.6 \mathrm{~dB}$. This is logical; when source and receiver are very close together, the primary contribution to the overall sound pressure level is from direct radiation, not from reflected sound. Further experimentation along these lines leads to the conclusion that errors in sound pressure level predictions will be minimal for $R / H$ less than one if absorption of the plane nearest the sound source is correctly specified.

The curves shown in Figs. 4 and 5 do not extend below an $R / H$ value of 0.15 or 0.2 , respectively, because that represents the minimum separation distance between the source and the receiver. If the source and the receiver are placed at the same height it is possible to extend the curves much farther downward as the source to receiver distance can be made as small as desired. When this is done, it is found that the curves become straight lines with a slope of two to one. This indicates a square law relationship between SUM and $R / H$ which is directly traceable to the square of the source-to-receiver distance in Eq. (17). Once this is realized, it is possible to extrapolate downward from a set of curves like those shown with sufficient accuracy without having to do the detailed calculations for the SUM.

For large values of $R / H$ (observer far from the source) the value of SUM approaches a limit. This limit is predictable. It can be shown that the length ratios in Eq. (17) approach unity at large values of $R / H$. Under these conditions, the summation becomes a geometric series for absorption values greater than zero. A simplified expression for SUM can then be derived:

$\operatorname{SUM}=2\left[\left(1-\alpha_{1}\right)\left(1-\alpha_{2}\right)\right]^{1 / 2} /\left\{1-\left[\left(1-\alpha_{1}\right)\left(1-\alpha_{2}\right)\right]^{1 / 2}\right\}$.

The validity of this expression can easily be checked by verifying values shown in Figs. 4 and 5 . It is also interesting to note that Eq. (23) indicates that curves for $\alpha_{1}=0.8, \alpha_{2}=0.2$, and for $\alpha_{1}=0.2, \alpha_{2}=0.8$, must approach the same value as $R / H$ gets large. This is also logical. At distances far from the source, all reflected sound will have undergone many reflections. Under these conditions the absorption of each plane is equally important. A more complete picture of the variation of SUM with absorption coefficients for fixed source and receiver heights may be gained by examining the figures in the Appendix.

There is one other important consequence of Eq. (23). Galaitsis and Patterson investigated transmission of sound between parallel planes having equal absorption coefficients. If their results are to be correct for large $R / H$ values, and if an attempt is made to use them for planes having unequal absorptions, it is evident that the mean reflection coefficient $(1-\alpha)$ used must be the geometric mean of the two reflection coefficients.

The variation of sound pressure level between parallel planes is indicated in Fig. 6. These curves were plotted for the source and receiver locations indicated in Fig. 4. Shown on Fig. 6 is a negative $6 \mathrm{~dB}$ per doubling line which corresponds to the sound pressure level which would result from direct spherical radiation. (This would also be the line for the case where both ab- 


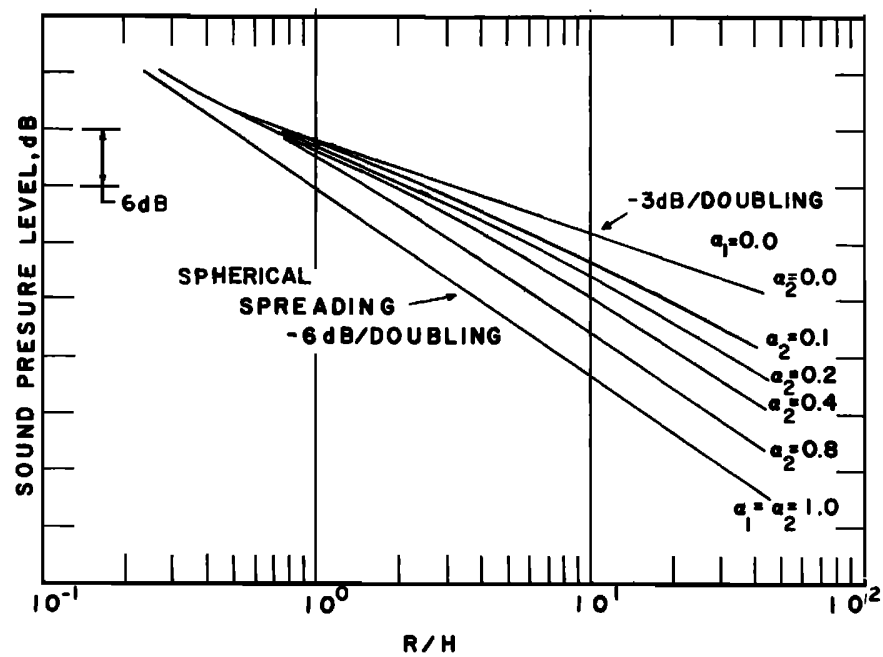

FIG. 6. Sound pressure level prediction between absorbing parallel planes.

sorption coefficients were one.) The remaining curves are for a hard "floor" $\left(\alpha_{1}=0.0\right)$ and for varying values of "ceiling" absorption. The latter curves were obtained by correcting from the spherical spreading curve by the amount $10 \log (1+$ SUM $)$. It will be seen that for both planes acoustically hard the sound pressure level decreases by $3 \mathrm{~dB}$ per doubling of distance. This is consistent with the fact that under these conditions the spreading of the sound would be approximately cylindrical at distances very much farther from the source than the plane separation distance.

It is also possible to check these results against those published by Galaitsis and Patterson by choosing equal plane absorption coefficients and identical source and receiver locations. When this is done the results of both investigations are in complete agreement. Some care must be taken in doing this. The independent pa- rameter chosen by Galaitsis and Patterson was the normalized horizontal distance $x / H$ rather than $R / H$. Thus the curves have a slightly different appearance when the receiver is close to the source.

The fact that SUM approaches a limit at large $R / H$ values for nonzero $\alpha$ values also means that all sound pressure level curves will ultimately become parallel to the spherical spreading loss curve, with a slope of $-6 \mathrm{~dB} /$ doubling of distance. The distance required to reach this condition will be very dependent, however, on the absorption of the planes.

The predictions of sound pressure levels inside a rectangular room can be obtained from the parallel plane results by using superposition. Such a room can be considered as three orthogonal sets of parallel planes, with three separation distances. If all distances are scaled in turn by each of these three $H$ values, it is possible to find three values of SUM (using, of course, the appropriate $\alpha_{1}$ and $\alpha_{2}$ values in each case). Since each of these SUM's included only the reflected energy, the total energy arriving at the receiver can be found by adding. Thus

$$
L-L_{s}=10 \log \left(1+\mathrm{SUM}_{1}+\mathrm{SUM}_{2}+\mathrm{SUM}_{3}\right)
$$

where $L_{s}$ is the sound pressure level at the receiver for spherical spreading of the sound. Applications of this approach to an imaginary square room with a ceiling height of $5 \mathrm{~m}$ is shown for two rooms in Fig. 7 . This was done to allow comparisons between the parallel plane results and those predicted by the conventional diffuse field method. For a room measuring $10 \mathrm{~m}$ on a side, it can be seen that the agreement is poor except very near the source: This is as it should be, since reflections from orthogonal planes become important in a small room such as this. The diffuse field equation results have generally been found valid in rooms of these dimensions. When the dimensions of the sides of the

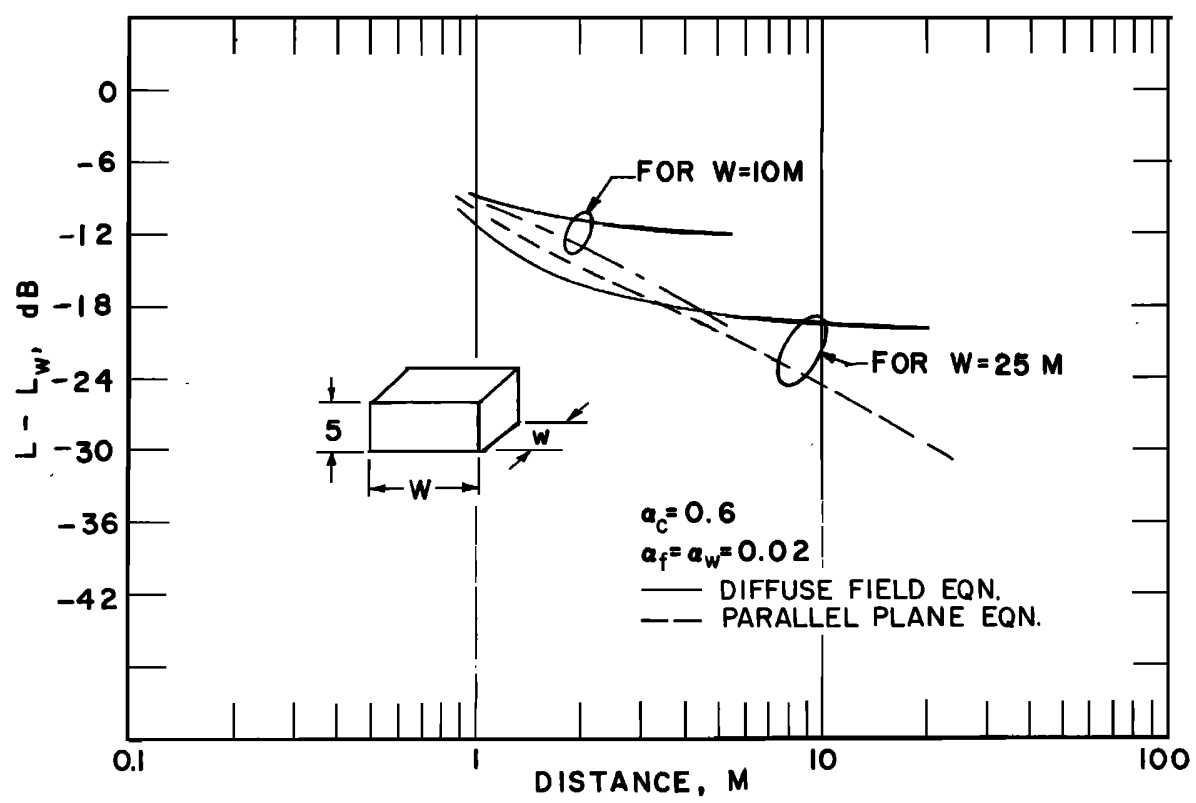

FIG. 7. Sound pressure level prediction in square rooms of the same height but different floor areas. 


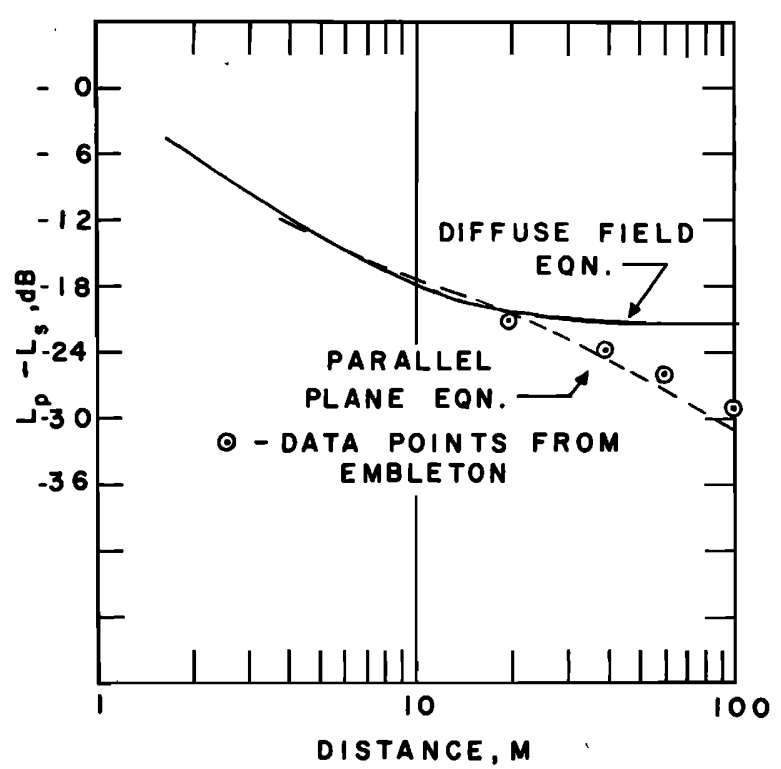

FIG. 8. Comparison of predictions using parallel plane method with data published by Embleton. ${ }^{4}$

room are increased to $25 \mathrm{~m}$, the agreement between the two methods is within about $1 \mathrm{~dB}$ for most locations near the source. As the distance from the source increases, however, the sound pressure level predicted using parallel planes is found to be much below that predicted by the diffuse field equations. It is next necessary to establish that the parallel plane method is the better prediction method in cases such as this.

Embleton $^{4}$ has reported an investigation in a large rectangular classroom. Although some details concerning his room are uncertain, there is sufficient information about it to make reasonable assumptions concerning the absorption values of the walls, floor, and ceiling. These assumptions will yield the diffuse field results shown by Embleton. When these assumed absorption values are used in the parallel plane approach, the results shown in Fig. 8 are obtained. The parallel plane approach appears to be the better prediction method in this room.

As a further test on the prediction capabilities of the parallel plane method, it was used to predict the sound

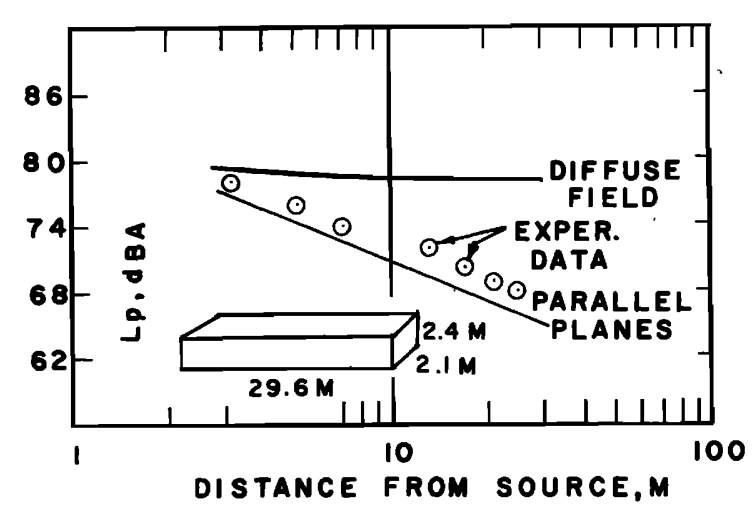

FIG. 9. Measured and predicted sound pressure levels in a long narrow hallway. pressure levels which would be produced by an ILG reference sound source in a long narrow hallway available on the VPI and SU campus. Comparisons were made with measurements, and the results are shown in Fig. 9. The parallel plane prediction method gave results which were consistently about 1.5 to $2 \mathrm{dBA}$ low. Much of this difference was felt to be attributable to the fact that general manufacturer's specifications were used for the sound power production of the ILG source, rather than individual calibration data. The trend in the sound field was correctly predicted. Results predicted by the diffuse field equation are also shown on this figure for comparison purposes. Because the assumptions on which that equation was derived are so badly violated, the equation gives predictions which are in error by a considerable amount.

This prediction method has been tested against other measured sound pressure level data, and against the sound pressure level predictions of a much more comprehensive geometric acoustics prediction program. In general, the results have been comparable to those presented here. In those cases where the dominant acoustical feature is a set of planes, and particularly where the measuring point was one or more plane separation distances from the source, the measured and predicted results agreed within about 1 to $2 \mathrm{~dB}$. This finding is also in agreement with the measurements of Galaitsis and Patterson.

\section{SUMMARY}

It has been shown that it is possible to predict sound transmission characteristics of absorbing parallel planes with relative ease. These results can then be used to predict the sound pressure level in those situations where the dominant acoustic feature is a pair of parallel reflecting planes. Such situations occur in large open plan offices, in factories, and in rooms where the proportions exceed $1: 1.5: 2$. The method predicts a drop in sound pressure level with distance which agrees with measured values.

\section{ACKNOWLEDGMENT}

This work was performed as a part of an effort to write computer programs capable of predicting noise levels in manufacturing plants. The work was supported by the National Science Foundation under Grant ENG74-00984A01.

\section{APPENDIX}

Included in this appendix is a series of curves (Figs. A1-A6) showing the results of the summation in Eq. (17) of the paper for a fixed receiver and source location, and for a range of values of the "floor" absorption $\alpha_{1}$. The availability of these curves will allow easy application of the parallel plane method of calculation for many cases of practical significance. 


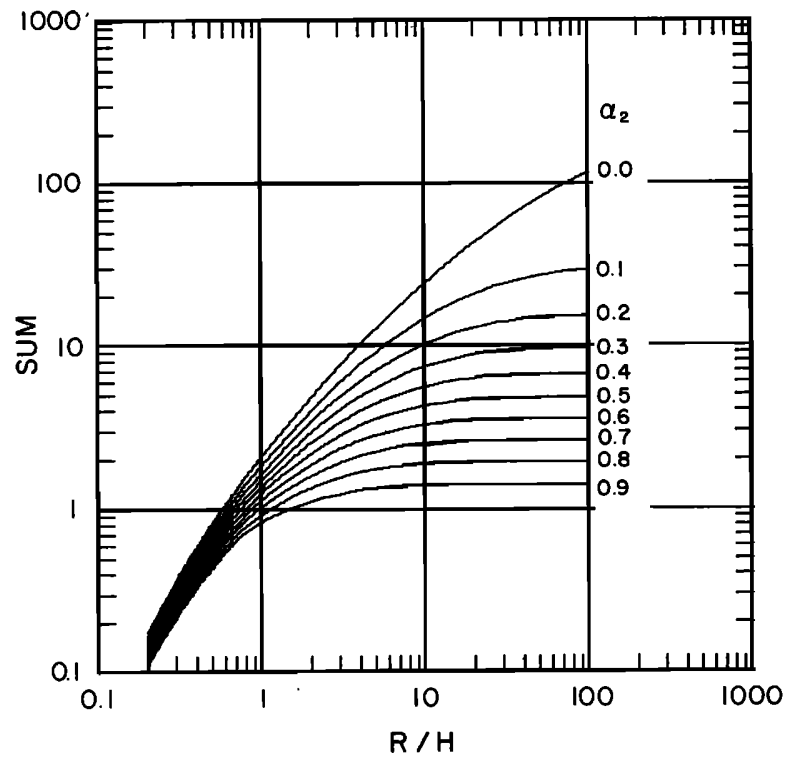

FIG. A1. Results of the summation for a floor absorption of $0.02 . y_{S} / H=0.2, y_{R} / H=0.4$.

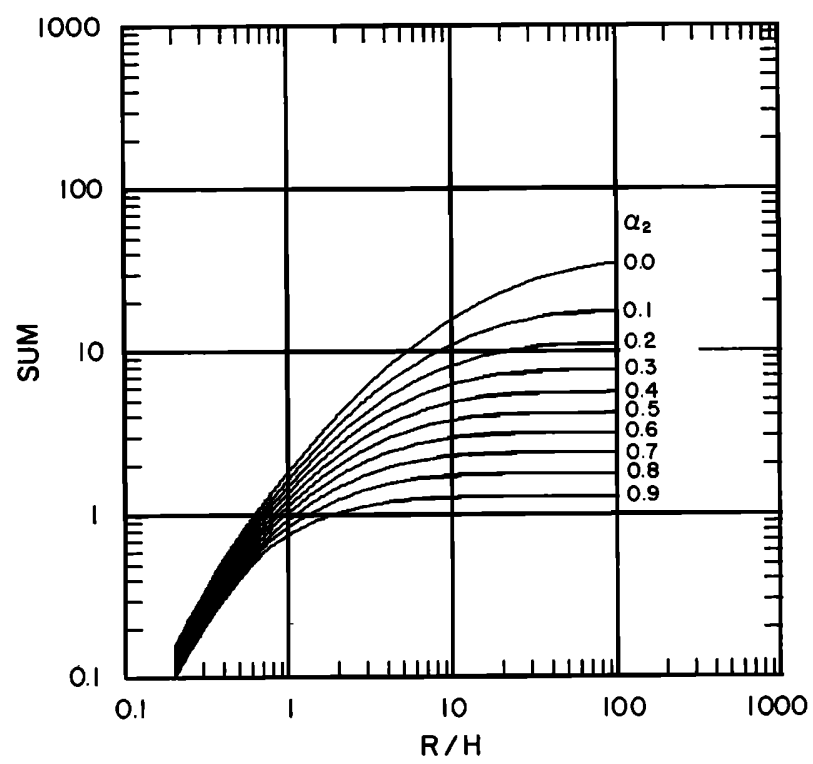

FIG. A2. Results of the summation for a floor absorption of 0.1. $y_{S} / H=0.2, y_{R} / H=0.4$.

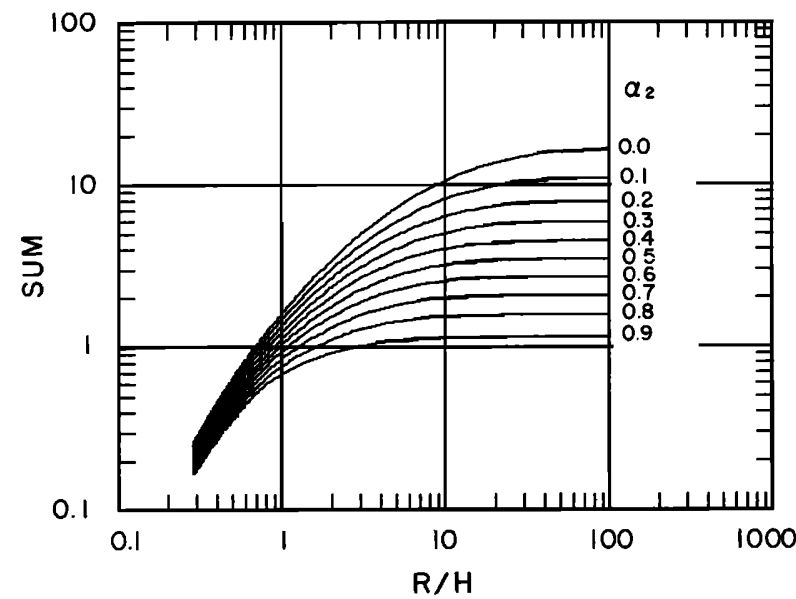

FIG. A3. Results of the summation for a floor absorption of $0.2 . y_{S} / H=0.2, y_{R} / H=0.4$.

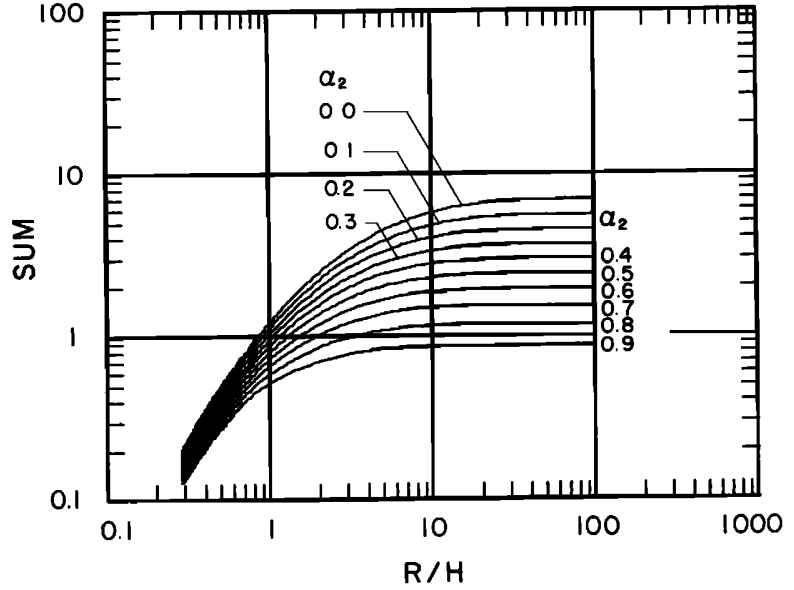

FIG. A4. Results of the summation for a floor absorption of 0.4. $y_{S} / H=0.2, y_{R} / H=0.4$.

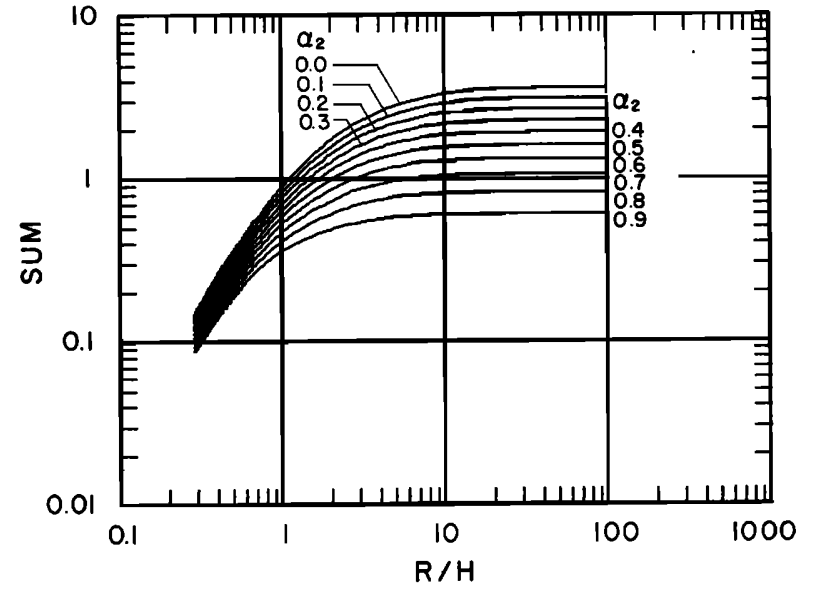

FIG. A5. Results of the summation for a floor absorption of 0.6. $y_{S} / H=0.2, y_{R} / H=0.4$.

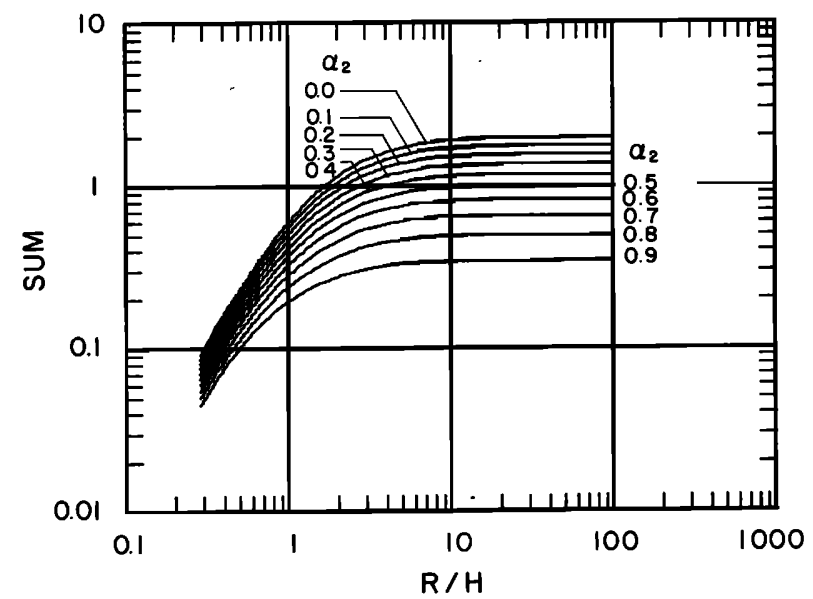

FIG. A6. Results of the summation for a floor absorption of 0.8. $y_{S} / H=0.2, y_{R} / H=0.4$. 
${ }^{1}$ G. Millington, "A Modified Formula for Reverberation," J. Acoust. Soc. Am. 4, 69-81 (1932).

${ }^{2}$ C. F. Eyring, "Reverberation Time in 'Dead' Rooms," J. Acoust. Soc. Am. 1, 217-241 (1930).

${ }^{3} \mathrm{~V}$. O. Knudson and C. M. Harris, Acoustical Designing in Architecture (Wiley, New York, 1950).

${ }^{4}$ T. F. W. Embelton, "Sound in Large Rooms," in Noise and Vibration Control, edited by L. L. Beranek (McGraw-Hill, New York, 1971), pp. 224-230.

${ }^{5}$ D. H. Lester, Jr., "Sound Pressure Level Predictions in Large Rooms," M. S. thesis, Virginia Polytechnic Institute and State University, Blacksburg, VA, May 1973.

${ }^{6}$ B. W. Mitchell, "Determination of Acoustic Ray Paths in Enclosed Spaces," M. S. thesis, Virginia Polytechnic Insti- tute and State University, Blacksburg, VA, February 1975.

${ }^{7} \mathrm{~B}$. R. Held, "The Determination of Sound Pressure Levels in Enclosed Spaces Using Geometric Acoustics," M. S. thesis, Virginia Polytechnic Institute and State University, Blacksburg, VA, January 1976.

${ }^{8}$ B. R. Held, B. W. Mitchell, C. J. Hurst, and L. D. Mitchell, "Sound Pressure Level Prediction in Large Rooms Using Geometric Acoustics," Proceedings, 1976 International Conference on Noise Control Engineering, Institute of Noise Control Engineering (1976), p. 259.

${ }^{9}$ A. G. Galaitsis and W. N. Petterson, "Prediction of Noise Distribution in Various Enclosures from Free-Field Measurements," J. Acoust. Soc. Am. 6, 848-856 (1976). 